\title{
Quelles contre-propositions ouvrières lors des restructurations industrielles en Wallonie de 1959 à 1984 ?
}

Stratégies de reconversion et paradigmes économiques

Workers' strategies and industrial restructurings in Wallonia from 1959 to 1984.

Reconversion options and economic paradigms

\section{Nicolas Verschueren}

\section{(2) OpenEdition}

\section{Journals}

Édition électronique

URL : http://journals.openedition.org/travailemploi/6182

DOI : $10.4000 /$ travailemploi.6182

ISSN : 1775-416X

Éditeur

DARES - Ministère du Travail

Édition imprimée

Date de publication : 15 mars 2014

Pagination : 21-35

ISSN : 0224-4365

Référence électronique

Nicolas Verschueren, «Quelles contre-propositions ouvrières lors des restructurations industrielles en Wallonie de 1959 à 1984 ? », Travail et Emploi [En ligne], 137 | janvier-mars 2014, mis en ligne le 01 janvier 2016, consulté le 02 mai 2019. URL : http://journals.openedition.org/travailemploi/6182 ; DOI : 10.4000/travailemploi.6182 


\title{
Quelles contre-propositions ouvrières lors des restructurations industrielles en Wallonie de 1959 à 1984 ? Stratégies de reconversion et paradigmes économiques
}

\author{
Nicolas Verschueren $\left.{ }^{*}\right)$
}

\begin{abstract}
Parcourant vingt-cinq ans de l'histoire industrielle de Wallonie, cet article offre une analyse des contre-propositions industrielles portées par les collectifs ouvriers lors des restructurations qui affectent leur secteur d'activité entre 1959 et 1984. Après, ou à côté de, l'opposition à la fermeture de leur lieu de travail, ils se sont inspirés de l'émergence ou de l'absence de nouveaux paradigmes économiques pour proposer leurs solutions originales de reclassement. Les notions de répartition économique régionale, de post-industrialisation, de tertiarisation, d'entrepreneuriat et d'économie de marché se trouvent réappropriées par les collectifs ouvriers pour formuler d'autres scénarii de reconversion, plus ou moins réalistes.
\end{abstract}

À l'automne 2011, la société Arcelor-Mittal annonçait l'arrêt de la phase à chaud de l'usine sidérurgique de Liège. Un peu plus d'un an après ce premier choc, une nouvelle restructuration était programmée condamnant à plus ou moins court terme le site sidérurgique liégeois, symbole de l'industrialisation de la Wallonie. Avec un recul d'un demi-siècle, l'histoire économique et sociale de cette partie de la Belgique semble se résumer à la notion de restructuration qui apparaît pour la première fois à la fin des années 1950. Ce trait volontairement grossi d'une histoire morose et sinistre de la Wallonie met en évidence la récurrence des restructurations d'entreprise depuis l'immédiat après-guerre jusqu'à aujourd'hui, longue période durant laquelle l'incertitude dans les décisions d'investissement dans l'industrie sidérurgique est devenue chronique (LombA, 2001, 2013). Les fermetures des mines de charbon, des ateliers de confection, des manufactures d'appareils électroniques ou des usines sidérurgiques ont pu être présentées comme un processus inéluctable lié à un impératif économique. La réduction de la consommation de charbon et l'internationalisation de certaines activités industrielles devenaient par ailleurs une occasion pour s'affranchir d'un emploi dangereux, éreintant et oppressant. L'essor de l'informatique, du secteur tertiaire et des moyens de communication ont contribué à sanctifier cette évolution vers une société postindustrielle, une ère nouvelle dans les étapes de la croissance économique dans les années 1960 (Rostow, 1962).

(*) Center for Transatlantic Relations, SAIS, Johns Hopkins University; niversch@ulb.ac.be

\begin{abstract}
À bien des égards, les luttes ouvrières menées lors des restructurations d'entreprises ont été montrées dans les médias comme des combats d'arrière-garde et des tentatives désespérées pour sauver un emploi condamné. Cette fatalité s'est insinuée progressivement dans les colonnes des quotidiens et les scripts des journaux télévisés, annonçant la fermeture, tel un événement inévitable et imprévisible au même titre que les catastrophes naturelles (CLARK, 2011). Pourtant, à l'image des bris de machine ou des émeutes alimentaires du début de la révolution industrielle (HoBsBawm, 1952; THOMPSON, 1971; JARRIGE, 2009), les conflits sociaux apparus lors des restructurations d'entreprises témoignent d'une prise de parole ouvrière recevant un écho dans l'espace public régional. Les formes de l'action collective - slogans, chants, arguments développés - et autres manifestations de la contestation formulées par les ouvriers dépassent la simple expression d'une colère pour révéler une compréhension certaine de leur situation économique et sociale.
\end{abstract}

La restructuration qui secoue la branche d'activité dans laquelle ils exercent leur profession pose inévitablement la question de leur devenir dans le système productif et des possibilités de réemploi qui s'offrent à eux. Or, reconversion industrielle d'un bassin d'emplois et reclassement professionnel individuel ne coïncident que trop rarement (BEATTY, Forthergill, 1996; Deshayes, 2010). Conscients de la situation économique de leur secteur, ces ouvriers et ouvrières sont amenés à entreprendre un processus de reconversion qui les éloigne d'un reclassement au sein d'un secteur en crise : ces initiatives tentent de concilier reconversion industrielle des territoires et reconversion professionnelle 
des individus (Hudson, 1994). Or, les deux processus suivent souvent des lignes parallèles qui voient l'implantation d'un centre commercial avec des offres d'emplois de caissières ou de caristes s'accorder difficilement avec l'engagement d'anciens tréfileurs ou de souffleurs de verre. Face à ces difficultés, les collectifs ouvriers et leur porte-parole sont rarement restés dans une simple opposition à la fermeture et les exemples de propositions ouvrières originales de reclassement abondent dans l'histoire de l'après-deuxième-guerre-mondiale en Wallonie. Qu'il s'agisse de paysans des Pouilles devenus mineurs, d'ouvriers de l'acier issus d'une longue lignée de sidérurgistes ou d'ouvrières de l'industrie textile sujette à délocalisations, leur perception des enjeux lors de la restructuration va au-delà de la sauvegarde de l'emploi pour envisager d'autres solutions de reclassement professionnel. Mais la mémoire collective garde peu de traces de ces propositions parfois restées à l'état de projets inaboutis ou utopiques.

Les expériences des restructurations telles que les vivent les ouvriers demeurent un territoire difficilement accessible pour l'historien, un champ d'investigation réservé aux sociologues et ethnologues (JAHODA et al., 1982; LAFAYE, 2005; LE Quentrec, Benson, 2005 ; Corteel, 2009; RoupnelFuENTES, 2011). Mais depuis une petite dizaine d'années, la recherche historique a emboîté le pas à ces derniers en s'inspirant des actions de sauvegarde de la mémoire ouvrière lors des restructurations d'entreprises dans les années 2000 (ClARK, 2011; FAVIER, 2009). Cela ne va pas soi, la difficulté de l'interprétation historique s'intensifiant avec l'éloignement temporel. Pister les parcours professionnels et les expériences de la restructuration des ouvriers de la mine, dans les années 1950, ou des piqueuses d'un atelier de confection, dans la Lorraine belge des années 1980, s'avère un exercice difficile au vu des rares documents dont l'historien dispose, à l'inverse des sociologues qui peuvent s'appuyer sur les documents de cellules de reclassement ou les associations d'anciens par exemple (MAZADE, 2010). Néanmoins, des traces, matériaux de l'historien, existent. Les chants ouvriers, les actions collectives ou les options discursives produites par les collectifs ouvriers, avec l'appui d'une expertise économique syndicale dans certains cas (LomBA, 2013), démontrent que les ouvriers ont une compréhension aiguë de leur situation économique et sociale. Dans ce contexte, les propositions de reclassement ou de reconversion qu'ils soumettent à leurs anciens employeurs, aux pouvoirs publics ou aux organisations syndicales illustrent plus qu'aucune autre les formes d'appropriation ouvrière des processus de restructuration.

Dans cet article, à partir de quatre restructurations situées dans des séquences historiques différentes (1959, 1976, 1977-1980, 1984), nous voudrions montrer l'intérêt de se pencher sur ces «contrepropositions ouvrières». Ces quatre cas d'étude ont été sélectionnés pour donner à voir des secteurs, époques et contextes industriels différents. Les dimensions sectorielle et régionale de la restructuration sont ici essentielles pour appréhender le rôle que les paradigmes économiques de la période ont pu jouer dans ces tentatives de reconversion impulsées par les ouvriers.

\section{Déclin charbonnier, migration et équilibre économique régional}

\section{Les mineurs italiens du Borinage et la crise charbonnière de 1959}

Le premier cas observé s'attache à montrer la manière dont les mineurs italiens du Borinage ont repris à leur compte la problématique de la «reconversion régionale» pour trouver du travail en dehors de la mine et s'assurer un reclassement dans leur bassin d'installation. En 1959, la crise charbonnière est un véritable test pour l'équilibre politique de l'État belge et, dans une certaine mesure, des institutions européennes (MILWARD, 1992; Verschueren, 2013a). La main-d'œuvre italienne, perçue comme une force de travail temporaire qu'on aurait pu rapidement rapatrier ou relocaliser, prend fait et cause pour cette dimension régionale de la lutte sociale.

En 1958, l'industrie du charbon est confrontée à la plus importante crise sectorielle de l'aprèsguerre. Les besoins en matières premières pour la reconstruction et la guerre de Corée, et le mythe du déficit charbonnier en Europe entretenu par les États-Unis dissimulent la crise de surproduction qui menace le vieux continent (PERRON, 1996). En Belgique, l'avenir du bassin charbonnier du Borinage est particulièrement sombre en raison de son caractère mono-industriel, de ses spécificités géologiques et d'une atmosphère de dépression qui semble ne pas avoir quitté la région depuis les années 1930. La crainte d'une explosion sociale est partagée tant par le gouvernement belge - la tradition de combativité des mineurs du Borinage est bien connue - que par la jeune Haute Autorité de la Communauté européenne du charbon et de l'acier qui doit faire face à la première crise sociale majeure de son existence (Verschueren, 2013a). Ce contexte explique l'extrême attention accordée à quelques milliers de mineurs à une époque où le chômage n'affecte guère les régions industrielles de la Communauté européenne (MiLwARD, 1992). La contestation ouvrière lors de l'annonce du plan de fermeture des puits du Borinage ne menace pourtant pas la stabilité sociale de l'ensemble des bassins industriels belges. A contrario, le faible soutien apporté par le reste du mouvement ouvrier sera un indice pour les responsables politiques 
belges et européens que le processus de restructuration dans l'industrie charbonnière peut désormais être accéléré.

Bien qu'ils ne prennent pas la forme insurrectionnelle tant redoutée, l'annonce du déclin programmé de l'industrie charbonnière provoque des remous non négligeables à l'intérieur du Borinage. Entre le 15 février et le 1 ${ }^{\text {er }}$ mars 1959, les grèves, manifestations, barricades et blocages de la circulation rythment le quotidien des localités minières du Borinage (GuBbels, 1962). Les demandes de reconversion et de reclassement $\mathrm{y}$ remplacent très rapidement le slogan «Non aux fermetures» omniprésent au début de la lutte sociale. Cette prompte résignation quant à la disparition de l'industrie charbonnière s'explique par l'absence de nationalisation des mines malgré le lancement d'une bataille du charbon ${ }^{(1)}$, la préservation des industries belges des dommages de guerre et l'importance quantitative de la main-d'œuvre italienne censée être temporaire. De fait, l'analyse statistique de la rotation de la main-d'œuvre dans l'industrie charbonnière montre que les ouvriers belges ont, dès les années 1930, tenté de trouver des échappatoires au travail minier dans le Borinage (BRUwiER, 1996).

Dans ce contexte, la problématique de la «reconversion régionale» portée par les élus locaux et discutée par des experts universitaires vise principalement à assurer l'avenir économique de la région. La fermeture des mines du Borinage se situe à la croisée des interrogations sur la politique économique des bassins industriels au lendemain de la découverte de la réalité régionale et de l'échec des migrations provoquées (Leboutte, 2008). L'échec du déplacement des mineurs du Centre-Midi en France vers la Lorraine en 1954 (REID, 1999) est tellement retentissant qu'un projet similaire n'est jamais publiquement évoqué pour le Borinage (Minon, Demet, 1956) (2). Le dépérissement de certaines régions au profit d'autres fait désormais l'objet de réflexions conséquentes dont le géographe Jean-François Gravier est le représentant le plus connu et dont les travaux sont suivis de près à la

(1) À l'image de la France en 1945, le gouvernement belge se lance dans une bataille de la production, une bataille qui est appelée à se gagner dans le secteur charbonnier. L'échec des projets de nationalisation lance un très mauvais signal aux mineurs belges déjà réticents à redescendre au fond en raison des conditions de travail dans des mines considérées parmi les plus dangereuses et vétustes d'Europe.

(2) En 1954, les Charbonnages de France avaient essayé de déplacer 5000 ouvriers du Centre vers les mines de Lorraine. Il s'agissait alors du plus grand projet de relocalisation de main-d'œuvre en France dans les années 1950. L'échec sera retentissant: seuls 387 ouvriers acceptèrent de tenter l'expérience. En outre, la campagne de dénonciation de ce projet par les communistes autour des thèmes de la déportation ou du service de travail obligatoire fut très efficace.
Communauté européenne du charbon et de l'acier (Ceca) ${ }^{(3)}$. La région du Borinage devient le laboratoire des sociologues belges qui multiplient les études sur la situation et l'avenir de cet emblématique bassin minier. Entre 1950 et 1962, trois études sociologiques sont menées pour dresser le bilan et l'avenir du bassin minier (4). Sur la base de ces réflexions, la Haute Autorité espère muer cette restructuration en première expérience de reconversion d'une vaste région industrielle. L'institution supranationale se présente comme le fer de lance de la propagation des notions de reclassement, de reconversion et de réadaptation.

\section{Le droit au reclassement sur place, même pour les ouvriers immigrés}

La question du « reclassement professionnel» sur place pour les mineurs privés d'emploi émane principalement des ouvriers, majoritairement italiens. Les syndicats CSC et FGTB (Confédération des syndicats chrétiens; Fédération générale du travail de Belgique) relaient cette revendication au détriment d'une politique de maintien de l'activité charbonnière et participent même à sa mise en œuvre. Comme le déclare Vital Décot, principal leader syndicaliste des mineurs de la région du Borinage : «Nous luttons pour le reclassement. Ce n'est pas pour les fosses que nous nous battons. Elles n'ont jamais fait que des cadavres avec nous ${ }^{(5)}$. » Avec la crise charbonnière est créé, en 1962, le Directoire charbonnier qui a notamment pour fonction de gérer les plans de fermeture et de fournir des programmes de reclassement pour les mineurs licenciés (EvalenKo, 1968, pp. 284-291). Cette institution, constituée de cinq personnes expertes dans le fonctionnement de l'économie du charbon ou dans les problèmes du travail qui lui sont liés, informe les organisations syndicales et

(3) Sur l'influence des géographes français pour la prise en considération de la problématique régionale, voir l'interview de Michel Bonnemaison, fonctionnaire à la Ceca en charge de la reconversion industrielle, par Yves Conrard et Ghjiseppu Lavezzi. Entretien avec Michel Bonnemaison par Yves Conrad et Ghjiseppu Lavezzi à Bruxelles le 12 février 2004 : http://www.eui.eu/HAEU/OralHistory/bin/CreaInt.asp?rc= INT704; consulté le 18 mars 2014.

(4) Ces enquêtes sociologiques sont conçues avec une finalité pragmatique, à savoir analyser la situation économique et sociale du Borinage, tirer les conséquences du déclin charbonnier et envisager des possibilités de reconversion régionale. La première étude d'après-guerre est publiée dans la Revue de l'Institut de sociologie en 1950. Une nouvelle enquête socioéconomique est menée quelques années plus tard par l'équipe du Centre d'économie régionale de l'Institut de sociologie de l'Université libre de Bruxelles (ULB) et publiée en 1957. Enfin, la dernière étude est réalisée par l'Institut de sociologie de l'ULB avec l'appui de l'Institut de recherches économiques du Hainaut et de la Société coopérative d'étude et d'assistance pour la reconversion économique des régions touchées par les fermetures de charbonnage: Les régions du Borinage et $d u$ Centre à l'heure de la reconversion, Bruxelles, Institut de sociologie Solvay, 1962.

(5) L'Est républicain, 19 février 1959, p. 12. 
les ouvriers des possibilités de reclassement dans les autres charbonnages du pays et des différentes allocations (belges et européennes) auxquelles ils ont droit pour leur réemploi, leur déménagement, etc. Si les organisations syndicales ne sont pas à l'origine du processus de reclassement des anciens mineurs, elles sont rapidement associées à la politique de réinsertion des travailleurs avant d'en devenir, dans certains cas, l'acteur principal comme nous le verrons pour les sidérurgistes d'Athus.

Dans cette reconfiguration économique régionale, la place des immigrés italiens n'est que peu évoquée. Après la signature de l'accord belgoitalien du 20 juin 1946, près de 80000 Italiens sont venus travailler dans les mines belges pour une durée de cinq ans (Morelli, 1988). La présence d'une main-d'œuvre immigrée devait faciliter le travail du gouvernement belge qui tablait sur un retour au pays de l'ordre de $15 \%$ et sur une mobilité importante de ces ouvriers entre les bassins charbonniers belges ${ }^{(6)}$. Malgré les retours en Italie et les quelques départs vers les autres régions industrielles, les mineurs italiens s'installent dans la région du Borinage (ANDRÉ, 1972). Loin d'être des acteurs passifs de cette évolution industrielle, ils sont le moteur de la révolte des mineurs dans le Borinage au cours du mois de février 1959, même si leur participation est perçue bien différemment dans la presse. Selon les journaux conservateurs, les révoltes sont essentiellement le fait d'agitateurs italiens, militants communistes et socialistes cherchant à déstabiliser le gouvernement démocrate-chrétien (7): ainsi, les commerçants du Borinage ne soutiennent la lutte des mineurs que par peur des représailles à l'encontre de leur boutique; et les voyageurs protestent contre le blocage de la circulation des trams par des Italiens. Pour la presse socialiste en revanche, il s'agit avant tout d'une révolte régionale de l'ensemble du bassin, caractéristique des communautés minières. De cette façon, l'italianité des très nombreux mineurs est passée sous silence et la question du reclassement de ces travailleurs «temporaires » évacuée.

Pourtant, ces Italiens craignent, à juste titre, que les mesures de reclassement pour les licenciés économiques bénéficient en priorité aux ouvriers belges comme c'est le cas pour les emplois dans la fonction publique. Dès lors, afin de garantir leur avenir professionnel dans la région, ils font entendre leur voix jusqu'à la Haute Autorité et font de la reconversion industrielle du Borinage le moyen de leur intégration dans la société belge et de leur sortie de l'activité charbonnière, qu'ils réprouvent

(6) Archives historiques de la Ceca, CEAB 7, n 306 , Fermeture de sièges au Borinage, Avis relatif à la lettre du ministre des Affaires économiques et des Classes moyennes, 27 novembre 1954.

(7) Le Journal de Mons et du Borinage, 19 février 1959. majoritairement ${ }^{(8)}$. Reprenant la thématique de la répartition géographique des richesses, la lutte pour le devenir de la région converge avec celui de leur sort d'immigrés venus travailler temporairement dans les mines de charbon.

La combinaison de la lutte pour l'emploi, de la défense des intérêts régionaux et des revendications des ouvriers immigrés est à l'époque inédite. Si l'échec des migrations provoquées est attesté (REID, 1999), la mobilisation des immigrés pour le devenir régional est inattendue. Cette mobilisation pour des aides publiques en faveur d'un plan de redéveloppement régional devient par la suite un trait récurrent des luttes sociales des mineurs de Belgique : les accords de Zwartberg en 1966 dans le Limbourg prévoient ainsi que chaque licencié dans l'industrie minière puisse être reclassé dans un autre site de production ou que la production du puits menacé soit maintenue aussi longtemps que des emplois de remplacement n'ont pas été créés. Bien qu'il s'agisse avant tout d'une déclaration d'intention, ce principe devient un référent des luttes sociales ultérieures; la proposition d'un plan de reconversion et/ou de reclassement de la main-d'œuvre peut alors constituer le socle de la revendication comme ce fut le cas lors de la fermeture de l'entreprise de construction métallique AngloGermain en 1967 dans le bassin du Centre ${ }^{(9)}$.

\section{Désindustrialisation, économie de services et reclassement dans le tertiaire}

\section{Des ouvrières en lutte contre la fermeture de l'usine Siemens en 1976}

Le deuxième cas d'étude s'intéresse à la fermeture en 1976 d'un atelier de réparation de relais téléphoniques Siemens, également situé dans le Borinage, symbolisant le déclin du travail manufacturier en Wallonie. Dans cet atelier travaillent majoritairement des ouvrières âgées en moyenne de 21 à 25 ans au moment de leur embauche ${ }^{(10)}$. L'examen de leur

(8) Lorsque les Italiens sont partis pour la Belgique, ils n'étaient bien souvent pas au fait du travail qui leur serait demandé. L'arrivée aux puits et la première descente étaient très impressionnantes et les tentatives de retour au pays se multiplièrent. Les conditions de travail étaient déplorables mais le contrat d'engagement de cinq années les empêchait de chercher un autre métier. La fermeture des puits leur offrit ainsi en quelque sorte une première grande opportunité de reclassement. (9) La fermeture de l'usine de matériel roulant AngloGermain est la première vaste restructuration d'une industrie dans la région de La Louvière, à l'exception du secteur charbonnier. L'accord de licenciement prévoit notamment le reclassement du personnel au sein d'autres entreprises spécialisées dans la construction de matériel roulant ainsi que, fait notable, l'assurance de commandes importantes auprès des usines de construction métallique.

(10) Institut d'histoire ouvrière économique et sociale, Centrale des métallurgistes de Belgique, section de MonsBorinage, farde 1, Liste du personnel, 1971. 
lutte nous intéresse à trois titres. Tout d'abord, elles ont fait de la problématique de la reconversion et de la responsabilité des investisseurs le centre de leur conflit. Ensuite, il s'agit alors de l'une des premières occupations d'usines par des ouvrières recevant une vaste publicité, qui plus est, face à une multinationale de la taille de Siemens. Leur action servira de modèle à plusieurs conflits sociaux mettant aux prises des ouvrières face à un employeur invisible ou despotique. Enfin, elles répondent au déclin de l'industrie manufacturière dans leur région par des propositions de reclassement vers des emplois de bureau.

Les années 1960 sont marquées du sceau de la poursuite de la production industrielle et manufacturière et surfent en cela sur la vague fordiste de l'après-guerre. Dans ce contexte, le Borinage a entamé sa reconversion avec pour ambition la reconstitution d'un tissu industriel moderne qui compense les pertes subies par la fermeture des mines de charbon. Parmi les nouvelles implantations figure une usine de réparation de postes téléphoniques Siemens qui ouvre ses portes en 1970. En échange d'aides publiques et de la garantie que l'administration belge lui achèterait du matériel, la multinationale allemande s'engage à mettre sur pied pendant plusieurs années une activité productive à forte innovation technologique ${ }^{(11)}$. Or l'usine du Borinage, dont l'activité principale se limite à la réparation de relais téléphoniques désuets, n'eut jamais cette vocation ${ }^{(12)}$. Outre l'attrait des aides à la reconversion, l'ancienne région charbonnière offre une main-d'œuvre féminine abondante et de nombreuses possibilités d'apports financiers en provenance des pouvoirs publics locaux tels que l'achat de matériel et la prise en charge de la formation du personnel. Lorsqu'elle n'a plus droit aux aides publiques cinq ans plus tard, l'entreprise allemande décide de fermer son usine du Borinage prétextant la non-obtention d'un contrat public au profit de la société Bell. L'entreprise compte jusqu'à 329 travailleurs en 1972 pour retomber à 209 au moment de sa fermeture quatre ans plus tard (CAPRON, 1977). Alors qu'elle a présenté son usine comme fondamentalement «sociale», la multinationale allemande devient alors le symbole de l'opportunisme entrepreneurial utilisant à court terme et à son seul profit les aides à la reconversion

(11) Pour l'ensemble du pays, la Confédération des syndicats chrétiens a chiffré le montant des aides publiques directes et indirectes à Siemens (achat de matériel, déduction fiscale, construction d'ateliers, formation professionnelle des ouvrières, etc.) entre 5,1 milliards et 7,6 milliards de francs belges (Centre d'animation et de recherche en histoire ouvrière et populaire, Archives du MOC Mons-Borinage, $\mathrm{n}^{\circ} 107$, conflit à Siemens, CSC, Stratégie des multinationales vis-à-vis des pouvoirs publics, le cas Siemens).

(12) Centre d'animation et de recherches en histoire ouvrière et populaire, Archives du Mouvement ouvrier chrétien de Mons-Borinage, farde $\mathrm{n}^{\circ} 107$, conflit à Siemens, Stratégie des multinationales vis-à-vis des pouvoirs publics, le cas Siemens. régionale et les contrats d'achats garantis par les administrations publiques.

Après l'échec des négociations entreprises par les syndicats pour éviter la restructuration, les ouvrières décident d'occuper l'usine à l'automne 1976 participant ainsi au raz-de-marée de conflits sociaux qui touche la Wallonie dans la seconde moitié des années 1970 (DEGÉE, 1980). Dans un premier temps, leur lutte est à la fois une source d'émancipation par rapport à leur milieu familial et une découverte du monde militant et associatif. Avec l'occupation de l'usine et l'intense conflit social contre l'imposante multinationale allemande, ces ouvrières participent de l'héritage de lutte des «gueules noires» tout en se positionnant en tant qu'acteur social et politique dont le rôle ne se limite pas au périmètre du foyer. Le lien avec la célèbre combativité des mineurs du Borinage trouve son origine dans certaines situations familiales où le grand-père, le père, le mari ou le frère ont connu l'univers particulier du travail charbonnier. Cette référence au tempérament frondeur des mineurs du Borinage est également un moyen de valoriser la lutte, d'autant que les ouvrières qui occupent l'usine et ainsi découchent du domicile familial, font l'objet de bien des médisances.

Cette occupation donne lieu à la création de chants, de pièces de théâtre et de spectacles qui seront diffusés dans les autres usines en occupation, dans certaines organisations ouvrières et dans les groupes hétérogènes de la Nouvelle Gauche(13) (Verschueren, 2011). Le conflit entre les ouvrières et la direction de Siemens permet surtout de créer un collectif des ouvrières licenciées. À l'image de nombreuses autres luttes d'ouvrières dans cette seconde moitié des années 1970, il ouvre la voie à un rapprochement entre les luttes pour l'émancipation féminine et celles pour l'emploi avec, par voie de conséquence, un recouvrement des problématiques de la domination masculine dans les foyers et à l'usine (MARUANI, 1979). Néanmoins, le rôle très important de certaines déléguées ouvrières et/ou syndicales (Liliane Ray chez Siemens, Paulette Henquinet chez Levi's) coupe en quelque sorte l'herbe sous le pied des mouvements féministes tels que les Marie Mineur ${ }^{(14)}$. Les débats sur la condition et l'émancipation des femmes, bien qu'évoqués lors de ces occupations d'usine, restent secondaires par rapport aux luttes menées pour la sauvegarde de l'emploi.

(13) La Nouvelle Gauche est un ensemble hétérogène d'organisations situées à l'extrême gauche du spectre politique. C'est lors de la grève des mineurs du Limbourg de 1966 que ces mouvements apparaissent pour la première fois accompagnés d'étudiants militants. Ils se caractérisent par un activisme politique très important, des modes d'actions inédits et variés et la recherche d'un contact immédiat avec la classe ouvrière.

(14) Les Marie Mineur est un mouvement féministe apparu en Belgique en 1970 en lien avec la création des Dolle Mina en Flandres. Les deux mouvements utilisent une icône ouvrière féminine du XIXe siècle comme référence historique. 


\section{Passer de l'atelier au bureau, l'espoir déçu des formations qualifiantes}

Dans un second temps, lorsque la fermeture du site semble inéluctable, se pose la question du reclassement professionnel. Pour Liliane Ray, ouvrière chez Siemens et déléguée syndicale de la Confédération des syndicats chrétiens (CSC), rencontrée le 8 décembre 2009, il est évident que "la production était dépassée dès le début, on servait juste à obtenir des contrats de l'administration pour Siemens et, nous, on pouvait se recaser nulle part, les filles d'atelier, on n'en voulait pas et il n'y avait plus d'emplois ailleurs ». La multiplication des restructurations en Wallonie, leur expérience au sein de cet atelier sans avenir et les faibles opportunités de reclassement qui s'offrent à elles, incitent les ouvrières à essayer d'obtenir les formations qualifiantes nécessaires à l'obtention de postes administratifs. Au lendemain du conflit social, le collectif d'occupation de l'usine est maintenu pour préserver les liens affectifs qui se sont noués. Il sert, en outre, à tenter d'obtenir une aide à la formation pour que les ouvrières puissent acquérir les compétences nécessaires afin de trouver un emploi dans l'administration ou un travail de bureau ${ }^{(15)}$. Mais cette volonté de reconversion vers le secteur tertiaire s'avère illusoire et leur manque de qualifications met un terme au projet collectif. L'idée a été soutenue par certains délégués actifs au sein de l'Institut supérieur de culture ouvrière (lié à la CSC). Mais deux circonstances historiques expliquent l'échec de ce projet de reconversion. La forte mobilisation des premières semaines s'est rapidement essoufflée, signant la fin brutale de l'occupation de l'usine à la veille des fêtes de fin d'année. Ensuite, l'implication des organisations syndicales dans le reclassement et la reconversion de la maind'œuvre ne commence véritablement qu'un an plus tard, en 1977, avec la fermeture de l'usine sidérurgique d'Athus et la création des cellules de l'emploi (voir infra). Par la suite, le rôle des organisations syndicales dans la gestion des restructurations en Belgique s'amplifie et se diversifie. Ainsi, trois ans plus tard, lorsque l'usine de confection de jeans Salik ferme ses portes à quelques kilomètres de chez Siemens, des experts syndicaux proposent diverses options aux ouvrières : coproduction avec des usines polonaises, développement d'activités de services à vocation sociale, puériculture, élevage de poulets, ventes au détail, etc. ${ }^{(16)}$.

(15) La Cité, 21 août 1978.

(16) Centre d'animation et de recherche en histoire ouvrière et populaire, Fonds Salik - L'Espérance, carton 8, Séminaire «Sans emploi », 8 et 9 septembre 1979. En lien avec l'occupation de l'usine Salik, des délégués syndicaux de la CSC avaient organisé une rencontre de deux jours avec des ouvrières afin de leur proposer des alternatives de reclassement possible en rapport avec leurs qualifications et la situation industrielle du Borinage.
Réparant des appareils destinés aux administrations belges, les ouvrières de Siemens s'approprient à leur niveau le discours sur la tertiarisation de l'économie. Les possibilités de reclassement dans l'industrie manufacturière se réduisent avec l'internationalisation d'une production à faible valeur ajoutée alors que les emplois de bureau offrent encore des opportunités de reclassement ${ }^{(17)}$. Près de trois ans après le choc pétrolier de 1973, les fermetures des petites entreprises manufacturières se succèdent en Wallonie (CAMmarata, Tilly, 2001). La prise de conscience du déclin manufacturier n'est que rarement suivie d'un projet de reconversion vers le secteur tertiaire. Si l'on prend l'exemple des restructurations dans l'industrie textile dans la seconde moitié des années 1970, on constate que les couturières des entreprises Farah, Salik, Daphica et Captain ne se sont pas lancées dans un processus de reconversion similaire. La lutte des ouvrières de Siemens a par contre inspiré de nombreuses autres occupations d'usines en Wallonie, principalement dans les ateliers employant une main-d'œuvre féminine. Le fait que l'usine où elles travaillaient ait au départ été destinée à assurer une partie de la reconversion régionale après les fermetures des mines les a confortées dans l'idée qu'un reclassement sur le long terme signifiait une transition vers un autre secteur d'activité. Elles ne sont pas les seules à faire ce constat. À la même époque, des sidérurgistes entreprennent une démarche analogue au lendemain de la fermeture de leur usine.

\section{La première vague de restructurations de la sidérurgie européenne}

\section{La fermeture de l'usine sidérurgique d'Athus en 1977}

Le troisième cas porte de nouveau sur l'industrie lourde et s'intéresse à la fermeture de l'usine sidérurgique d'Athus en 1977, première restructuration totale dans le secteur de l'acier. L'usine d'Athus, située aux confins méridionaux de la Belgique, est alors l'employeur principal de la province du Luxembourg et le symbole de son activité économique à une époque où les responsables politiques et la population se considèrent comme les laissés-pour-compte de la Belgique. L'occupation de l'usine, la violence de certaines actions militantes, les tensions diplomatiques entre la Belgique et le Luxembourg lors de ce conflit mettent cette restructuration sur le devant de la scène médiatique en pleine période estivale, alors que les journaux peinent à remplir leurs colonnes. À la veille d'une

(17) Amsab - Instituut voor Sociale Geschiedenis, Archives Émilienne-Brunfaut, dossier relatif à un projet pilote de formation professionnelle des ouvrières de Siemens, 1977. 
succession de restructurations industrielles lourdes, l'économie nationale belge avait encore les reins assez solides pour injecter d'importantes sommes d'argent dans la relance industrielle régionale. Aussi, la médiatisation du conflit et le caractère pionnier de la restructuration expliquent-ils l'importance des moyens et des efforts consentis pour sortir les sidérurgistes de la crise sectorielle et régionale.

L'usine sidérurgique d'Athus est atypique dans le panorama industriel belge tant du point de vue géographique qu'historique. Créée en 1872, l'usine a prospéré parallèlement aux industries de Lorraine et du Grand-Duché du Luxembourg grâce à l'exploitation du minerai de fer lorrain. Ce positionnement géographique, pertinent à la fin du XIXe siècle, perd de son intérêt un siècle plus tard avec le développement de la sidérurgie maritime et la croissance de la concurrence mondiale pour les produits bruts ou semi-finis (MÉNY, WRIGHT, 1985). Un siècle après la construction du premier haut fourneau à Athus, l'usine sort du giron du capitalisme financier belge pour être associée à l'usine luxembourgeoise de Rodange et ainsi devenir la Minière et Métallurgie de Rodange-Athus. Ces deux sites, séparés de quelques centaines de mètres et qui se font concurrence depuis une centaine d'années, vont partager un bref avenir commun entre 1973 et 1977.

À l'été 1976, la situation commerciale de l'usine se détériore brusquement avec la chute de la demande de ronds à béton dont la production, bien que peu rentable, est une des spécialités d'Athus. Un plan d'austérité visant à réduire les pertes de l'entreprise est mis en place mais les prix des ronds à béton poursuivent leur effondrement en raison de la dépression qui affecte le marché de la production sidérurgique. Le sort de l'usine est scellé. L'annonce de la fermeture tombe le 26 juillet 1977 , provoquant l'occupation de l'usine et une série d'actions de protestation diverses et médiatiques : opérations «ville morte», occupation de l'ambassade du Grand-Duché du Luxembourg à Bruxelles ou encore mise à sac d'une banque locale. Durant tout l'été 1977, les sidérurgistes d'Athus sont au centre de la vie politique et médiatique de la Belgique; ils deviennent un des symboles du regain de la conflictualité ouvrière dans les années 1970 et, plus largement, de la violence contestataire chez les sidérurgistes de la région (VIGNA, 2004).

Première restructuration majeure de la sidérurgie européenne, l'extinction des hauts fourneaux d'Athus est annonciatrice de la crise de l'acier sur le point de sévir en Europe (Goffin et al., 1981). La province du Luxembourg perd son plus gros employeur du secteur privé et doit reclasser près de 2000 sidérurgistes alors que de restructurations sidérurgiques balaient toute la région des trois frontières ${ }^{(18)}$. Le gouvernement belge se lance alors dans une tentative de reconversion de cette région sinistrée avec la mise en œuvre de deux programmes inédits : une cellule de l'emploi et une société de diversification économique. La cellule de l'emploi a pour but de garantir un revenu dégressif sur trois ans pour le personnel licencié(19). Le système de dégressivité de ces allocations supplétives n'est pas nouveau : il a en partie été utilisé, avec le soutien des institutions européennes, lors des premières vastes restructurations charbonnières de 1959. La cellule de l'emploi représente un cas unique, innovant, de gestion d'une restructuration, d'autant plus significatif que son fonctionnement quotidien est géré par quatre délégués syndicaux et vingttrois anciens sidérurgistes. Outre le versement des allocations, elle sert de centrale de formation et de placement pour d'anciens sidérurgistes d'Athus avec des résultats en demi-teinte (VERSCHUEREN, 2013b). Elle crée d'ailleurs un précédent fameux, par la suite réutilisé dans plusieurs restructurations profondes: sidérurgie de Jemappes dans le Borinage et soieries Fabelta à Tubize (TILly, 2007). Selon Gérard Thiry, ancien ouvrier d'Athus et délégué syndical CSC :

«Les ouvriers qualifiés de moins de 40 ans ont été vite reclassés. Mais pour les ouvriers qui travaillaient au blooming (20), à la fonderie, au fil à béton, ils n'étaient plus qualifiés. On n'en voulait plus nulle part. C'est aussi pourquoi on s'est battu pour obtenir un important volet social. »

(Entretien avec Gérard Thiry, sidérurgiste à Athus, 12 décembre 2011.)

À la date de la restructuration, plus de la moitié des sidérurgistes a plus de 45 ans; les possibilités de reclassement sont en outre des plus limitées dans un bassin d'emplois saturé par les ouvriers licenciés du secteur de l'acier. Un tiers d'entre eux - des ouvriers atteignant ou dépassant l'âge de 55 ans dans les trois ans qui suivent la fermeture de l'usine - a obtenu une préretraite, système d'accompagnement particulièrement utilisé en Belgique (Houseman, 1991). Pour les autres, les postes disponibles sont de plus en plus rares dans la sidérurgie en crise; quant aux nouvelles entreprises venues s'installer dans la région, elles n'embauchent que peu d'anciens sidérurgistes.

L'autre dispositif public, la société de diversification belgo-luxembourgeoise, a pour objectif d'assainir le site de l'usine (aménagement d'espaces

(18) Ainsi est dénommée la région sidérurgique englobant les localités française, belge et luxembourgeoise d'Athus, Longwy et Rodange-Pétange.

(19) Cette somme dégressive est ajoutée aux allocations de chômage afin que l'ouvrier licencié touche un revenu équivalant à $100 \%$ de son dernier salaire la première année, $90 \%$ la deuxième année et $80 \%$ la troisième année de chômage.

(20) Le blooming est une étape de la fabrication de l'acier qui consiste à sortir des lingotières des produits d'acier semi-finis. 
verts, destruction des friches industrielles, éradication des crassiers) et de redévelopper une activité économique dans la région. Quelques entreprises s'installent sur l'ancien site grâce aux aides à la reconversion et une trentaine d'emplois sont créés pour les anciens ouvriers de l'usine d'Athus. Ces sociétés, mal constituées, profitant des subventions publiques, sont liquidées quelques années voire quelques mois à peine après leur création. Sur les 498 travailleurs qui ont retrouvé un emploi en 1980, 163 ont décroché un poste dans des organismes publics, dans l'armée ou dans l'enseignement. Une centaine d'autres sont recrutés par des entreprises soutenues directement ou indirectement par les pouvoirs publics ${ }^{(21)}$. La relance économique régionale semble avoir fait long feu : les mesures prises par le gouvernement belge ont davantage permis de soutenir financièrement les ouvriers licenciés que de mettre sur pied une nouvelle dynamique industrielle.

\section{De la production aux frontières à l'échange dans le Marché commun}

Néanmoins, la relance a lieu quelques années plus tard avec le Pôle européen de développement (PED) considéré à l'époque par Jacques Delors comme un laboratoire de l'Europe à l'échelle un millième. L'idée directrice du pôle est de transformer ces trois culs-de-sac nationaux que sont la Lorraine belge, la Lorraine française et le sud-ouest du Luxembourg en un centre économique européen leur permettant de surmonter la crise sidérurgique (22). Concernant plus directement l'usine d'Athus, une ingénieuse hypothèse de reconversion est imaginée par huit sidérurgistes d'Athus emmenés par Paul Viot, ancien chef du service traction ${ }^{(23)}$. Très rapidement, ils perçoivent le potentiel géographique d'une région bénéficiant d'un réseau de voies de communication hérité du passé industriel et située au cœur du marché commun au point de rencontre de trois États membres (24). À l'instigation de Paul Viot, l'idée émerge de mettre à profit leur expérience professionnelle pour développer une entreprise

(21) L'Avenir du Luxembourg, 30 juillet 1980.

(22) Les résultats du Ped varient fortement d'un pays à l'autre. Le Sud-Luxembourg belge doit beaucoup à la réussite économique du Grand-Duché. L'épisode malheureux de Daewoo en Lorraine française a également été vécu en Belgique sur une plus petite échelle avec des investisseurs opportunistes : ARC, Lord Marine, Ocribel, etc.

(23) Ce département de l'usine était chargé de la circulation des trains de manœuvre (lingotières et autres contenants de métal en fusion ou solide) à l'intérieur de l'usine.

(24) Sur les luttes et les expériences traditionnelles et originales dans la région longovicienne, voir: HAYES I. (2013), «Les limites d'une médiation militante. L'expérience de Radio Lorraine Cour d'acier, Longwy, 1979-1980», Actes de la recherche en sciences sociales, $\mathrm{n}^{\circ}$ 196-197, pp. 84-101; Azzaou B., Norriel G. (1980), Vivre et lutter à Longwy, Paris, Maspero; Charasse D. (1980), Lorraine Cæur d'acier, Paris, Maspero. de transport de containers entre les grands ports de la mer du Nord et un espace géographique qui deviendra la future grande région Saar-LorLux. Ce projet inattendu tourne à la success story d'une petite société, Terminal Container, qui de 2000 containers traités en 1979, devient le plus grand port sec de la mer du Nord avec un acheminement annuel de 120000 containers en 2006. L'entreprise recrute vingt-sept anciens sidérurgistes d'Athus et voit le départ du dernier « recasé » en $2003^{(25)}$.

À l'image des conflits sociaux examinés précédemment, la stratégie des huit travailleurs d'Athus repose sur un triple constat. Premièrement, la possibilité de retrouver de l'emploi dans un secteur sidérurgique en crise est minime, voire quasi nulle, pour des travailleurs âgés qui pressentent la possible extinction de leurs hauts fourneaux depuis le milieu des années 1960. Deuxièmement, la reconversion dans l'industrie lourde ou manufacturière locale paraît illusoire ou promise aux ouvriers plus jeunes disposant de diplômes plus récents (BASTin, 1977). En dernier lieu, ces ouvriers, qui ont participé à la première usine sidérurgique transfrontalière, ont une expérience pratique du fonctionnement $\mathrm{du}$ marché commun, ne serait-ce que par le transfert des marchandises et des personnes entre la Belgique et le Grand-Duché tout au long des six semaines de conflits. Leur discernement précoce anticipe au fond les projets européens de reconversion qui fleurissent dans les années 1980. La société créée par les anciens d'Athus devient un exemple et un point de fixation pour une régénération économique dont le marché commun et le transfrontalier devaient être les pierres angulaires (Ait Oumeziane, 2000). Pour l'ancien chef du service traction de l'usine sidérurgique, l'avenir d'Athus et de sa région passe par la prise en considération de la situation géographique idéale du Sud-Luxembourg au cœur de la Communauté européenne. Au début des années 1980, l'échec de plusieurs tentatives d'implantation d'entreprises manufacturière et de transformation métallique (Arc, Ocribel, Lord Marine, etc.) soutenues par les pouvoirs publics semble confirmer son intuition. À partir de ce constat, une forme de symbiose se développe entre les initiatives publiques locales et européennes de reconversion industrielle et l'essor progressif du terminal de containers. L'expérience de ces ouvriers au service traction de l'usine leur a permis de pressentir une reconversion axée autour de l'échange et de la logistique. Ils se sont ensuite appropriés le marketing public à destination des investisseurs potentiels, qui fait de la région des trois frontières, le carrefour des échanges commerciaux du Marché commun.

(25) Le Soir, 16 septembre 2003. 


\section{Contractions budgétaires et initiative privée}

\section{L'usine Levi's à Arlon en 1984 ou la fin du secteur textile en Belgique}

Le dernier exemple, qui illustre pour la Wallonie l'évolution des stratégies ouvrières lors des restructurations industrielles au regard des mutations du contexte et du discours économique, est consacré à la fermeture de l'usine de confection Levi's à Arlon dans l'extrême sud de la Belgique en 1984. L'histoire de cette usine et du conflit social qui fait suite à l'annonce de sa restructuration est mal connue. Peu d'archives ont été conservées, les ouvrières en lutte n'ont été que faiblement médiatisées alors que le contexte économique ne favorisait pas l'éclosion d'un élan social émancipateur tel celui apparu dans la seconde moitié des années 1970 (MoLitor, 1978).

Dernier grand atelier dans le secteur de la confection en Wallonie, sa disparition clôt un cycle de restructurations qui frappent cette industrie depuis plus de dix ans. Alors que les utopies sociales perdent de leur superbe et que le gouvernement applique une politique néolibérale, marquée par un gel des revenus incluant l'indexation automatique des salaires, l'examen de la fermeture de l'usine Levi's apporte un éclairage saisissant sur le développement d'une initiative ouvrière destinée à attirer les investisseurs potentiels et donner la priorité aux ouvrières licenciées de Levi's lors de futurs recrutements. La société américaine installe un atelier de confection à Arlon en 1970, profitant de l'importante réserve de main-d'œuvre féminine dans une région où les conflits sociaux sont peu nombreux et faiblement radicalisés. Comme dans tous les ateliers de confection, les conditions de travail sont difficiles, rythmées par une cadence infernale où chaque dépassement des quotas de production par poste dégage quelque complément de salaire. Une ouvrière explique :

«Je suis devenue au fil des ans, une spécialiste de la pose des fermetures éclair, même si je suis polyvalente. Je parviens à poser entre 11000 et 13000 "tirettes (26)", par jour [sic]. Le rythme est soutenu et je n'ai jamais eu beaucoup de temps pour bavarder ou lever le nez de ma machine à coudre. J'éprouve presque du soulagement à savoir que je vais plus travailler là...»

(Entretien avec Francine Jacob, deuxième ouvrière européenne en productivité de Levi's, in Libelle, $\mathrm{n}^{\circ} 81,18$ décembre 1984.)

Au 27 septembre 1984, l'usine compte 397 travailleurs, presque exclusivement des femmes d'une moyenne d'âge de 31 ans et au faible niveau de qualification (27). Une fois encore, les possibili-

(26) Belgicisme : fermetures éclair.

(27) Centre d'animation et de recherche en histoire ouvrière et populaire, archives de la CSC du Luxembourg conservées à Ciney, boîte 320, Lettre de Michel Hanson à Jean Devile, président de la Fondation André-Oleffe, 22 septembre 1987. tés de reclassement sont minimes dans le secteur manufacturier. L'atelier de Levi's à Arlon est la plus grande entreprise de confection de Wallonie au début des années 1980 et occupe $10 \%$ de la maind'œuvre totale de ce secteur. Quatre années après la restructuration, 225 ouvrières sont toujours au chômage. Paulette Henquinet, ouvrière chez Levi's et fer de lance de la mobilisation, explique le désarroi des jeunes femmes en ces termes :

"Nous étions surprises de la fermeture, l'usine marchait bien, on avait la même productivité et nos jeans avaient la meilleure qualité. Pourquoi fermer l'usine d'Arlon et pas celles de France ou de Flandres?»

(Entretien avec Paulette Henquinet, ouvrière et déléguée syndicale CSC chez Levi's, 18 août 2011.)

L'esprit combatif est pourtant bien présent. Ainsi, à l'annonce de la fermeture de l'usine, les ouvrières entament rapidement un bras de fer avec la direction belge de Levi's. Les tensions sont devenues très fortes à cause d'un manager flamand très peu apprécié des ouvrières. Lors des négociations, la direction menace ainsi de donner les noms «des mauvaises ouvrières » au nouvel investisseur en cas de remous (28). Le conflit social rappelle des formes culturelles de la protestation qui ont marqué le paysage socio-économique de la Belgique dans les années 1970 (Bettens, GeERKens, 2010).

Mais l'heure n'est plus aux occupations d'usine, la radicalité dans la lutte ouvrière étant peu répandue dans le mouvement ouvrier du Luxembourg belge. En outre, l'éloignement géographique est un obstacle conséquent tant pour la venue de militants extérieurs que pour la circulation de l'information. À vrai dire, peu nombreuses sont les personnes véritablement informées sur la situation des ouvrières de Levi's. Bien qu'aucun projet d'autogestion n'ait jamais été explicitement formulé et soutenu, la question est abordée notamment lors de l'organisation d'une visite des Textiles d'Ere en 1986, entreprise autogérée par des ouvrières dans la région de Tournai. Une cinquantaine d'ouvrières de Levi's lancent alors une autoproduction de jeans dans un entrepôt mais elle s'essouffle très vite par manque de rentabilité et de discipline ${ }^{(29)}$. L'utopie autogestionnaire laisse place à un pessimisme croissant sur l'avenir de l'industrie manufacturière

(28) Centre d'animation et de recherche en histoire ouvrière et populaire, archives de la CSC du Luxembourg conservées à Ciney, boîte 321, rapport du Conseil d'entreprise extraordinaire, 17 octobre 1984. Dans le procès-verbal modifié de cette réunion, la référence aux mauvaises ouvrières a été supprimée (le brouillon du premier procès-verbal, présent dans les archives, comportait la mention biffée et l'information a été confirmée par Paulette Henquinet, déléguée syndicale CSC).

(29) Pour un récit de l'intérieur sur la gestion au quotidien d'une usine en autoproduction par des femmes en 1977 en Angleterre, voir l'ouvrage de Judy Wajcman (1983), Women in control: dilemmas of a workers' co-operative, New York, St. Martin's Press. 
marquée par la rigueur néolibérale des deux législatures libérales-chrétiennes des ministres Jean Gol et Wilfried Martens. Cette période caractérisée par une réduction des dépenses publiques et par une forte modération salariale assomme littéralement les organisations ouvrières qui en gardent encore aujourd'hui un souvenir amer.

\section{La collectivisation des indemnités pour "attirer" les repreneurs}

Pour les ouvrières de Levi's en 1984, les options économiques et professionnelles sont inexistantes. $\mathrm{Si}$ les perspectives de reconversion régionale, de l'économie de services et de l'échange ont pu fournir des alternatives crédibles aux mineurs, aux ouvrières de Siemens et aux sidérurgistes licenciés, aucune option de ce genre ne s'offre aux «filles de Levi's» comme on les appelle alors. Le monde politique est dans une phase de désengagement, le «droit au reclassement» n'est plus pensé en termes collectifs mais sous l'angle de l'employabilité individuelle et le mouvement ouvrier entre dans une phase d'invisibilité médiatique. Les ouvrières de Levi's n'ont donc pas l'opportunité de bénéficier d'une cellule de l'emploi et sont, pour la plupart, bien trop jeunes pour obtenir un plan de préretraite sur une large échelle. Ce sont surtout les politiques d'austérité des années 1980 qui expliquent l'absence d'une cellule de l'emploi suite à la suppression des indemnités de formation professionnelle, qui offrent un complément aux allocations de chômage, des allocations de reconversion (HEGALE, LAYON, 2002) (30). En somme, il reste aux ouvrières de Levi's à choisir entre se résigner à un combat ouvrier qui serait perdu d'avance et se lancer dans l'initiative privée comme autre forme de réussite professionnelle, deux marqueurs forts des années 1980 en Belgique.

En l'absence d'un acteur politique et syndical soucieux de leur reclassement dans une région où l'emploi manufacturier féminin est quasi inexistant, les ouvrières créent une association à but non lucratif dont la principale activité est de gérer les indemnités de licenciement et de faire pression pour recevoir des garanties de réemploi dans toute entreprise qui viendrait s'implanter dans la région ${ }^{(31)}$.

(30) Il existe néanmoins dans le Sud-Luxembourg, à Weyler, une cellule de formation-reconversion créée en juin 1984, censée se substituer à la société de diversification belgo-luxembourgeoise créée au lendemain de la restructuration de l'usine sidérurgique d'Athus. L'existence de cette cellule de formation-reconversion explique l'absence d'une structure dédiée à l'accompagnement des licenciements chez Levi's mais les ouvrières n'ont pas pu en profiter longtemps, car elle s'arrête après une année de fonctionnement.

(31) Le délégué socialiste de la FGTB, Joseph Binet, souhaite obtenir des primes de licenciement importantes et n'accorde que peu d'attention aux propositions alternatives telles que d'éventuelles tentatives d'autogestion. Si la CSC y est davantage ouverte, c'est surtout la personnalité de Paulette Henquinet, déléguée CSC, qui est le moteur de la mobilisation.
L'intérêt pour ce qui est appelé le volet économique de la restructuration est bien une spécificité de ce conflit social. Une des revendications principales des ouvrières mobilisées est l'obtention du titre de propriété de l'atelier par les pouvoirs publics locaux, malgré l'opposition de la direction américaine, afin de disposer d'un bien immobilier permettant d'attirer un éventuel investisseur et qui leur servirait de gage pour leur reclassement. Cette revendication est clairement transposée dans le compromis de vente du bâtiment et du terrain de l'usine Levi's aux pouvoirs publics, sous l'appellation Idélux, dans son article 7 : «Idélux s'engage à tout mettre en œuvre afin d'installer ou de faire installer dans le bien vendu une entreprise [...] qui recrutera son personnel par priorité auprès du personnel employé par Levi's dans le bien vendu et qui sera licencié par Levi's, dans le cadre de la fermeture de l'usine d'Arlon ${ }^{(32)}$.» En outre, les représentants syndicaux de la FGTB et de la CSC se sont engagés auprès de la direction américaine à éviter tout acte de sabotage sous réserve que leur soit proposé un volet économique au plan de restructuration que la direction de San Francisco souhaite sans conflit. Les ouvrières obtiennent une indemnité de licenciement équivalant à $90 \%$ de leur salaire la première année et à $70 \%$ la deuxième année (maintien de $90 \%$ pour les ouvrières «cheffes» de ménage).

Mais surtout, une part du montant obtenu est collectivisée pour être utilisée en tant que fonds d'investissement. Cette initiative originale est critiquée par le syndicat socialiste(FGTB) et considérée comme une «frivolité» par les pouvoirs publics locaux. De telles prises de position recouvrent en grande partie le clivage politique opposant socialistes et démocrateschrétiens, la FGTB et l'intercommunale locale étant sur la même longueur d'onde. Ainsi, le principal représentant syndical FGTB dans la région, Joseph Binet, déclare aux ouvrières à propos de ce fonds d'investissement destiné à attirer de potentiels investisseurs : «Ne vous faites pas d'illusion, le promoteur peut très bien ne pas vous engager ${ }^{(33)}$.» Les indemnités atteignent un total de 51 millions de francs belges dont 9 millions doivent être versés au fonds d'investissement ${ }^{(34)}$. L'Association des ouvrières de Levi's a en outre conclu une convention avec les pouvoirs publics locaux pour être tenues informées de l'évolution des projets d'investissement et recevoir un traitement préférentiel. À ce titre, elles engagent un avocat qui intervient lorsque la société italienne Ferrero envisage de s'installer dans la région en 1989. Dans un premier temps, il a été contacté pour

(32) Centre d'animation et de recherche en histoire ouvrière et populaire, archives de la CSC du Luxembourg conservées à Ciney, boîte 196, compromis de vente entre Levi Strauss and Co Europe S. A. et l'Association intercommunale d'équipement économique «Idélux».

(33) La Meuse, 24 et 25 octobre 1984, p. 3.

(34) Soit près de 15000000 d'euros actuels en indemnités de licenciement. 
défendre les ouvrières contre la multinationale du jean et pour représenter les intérêts du collectif des ouvrières licenciées auprès des institutions locales. Avec l'arrivée possible de Ferrero, l'Association des ouvrières licenciées se mobilise immédiatement et fait pression sur les responsables politiques locaux pour qu'ils respectent leurs engagements et qu'elles obtiennent d'être prioritaires dans le recrutement du personnel lors de l'implantation de l'usine à Arlon. Ainsi, 106 ouvrières de Levi's sont recrutées dans les deux années qui suivent l'arrivée de Ferrero; il reste alors 76 ouvrières sans emploi(35). L'éloignement géographique du conflit chez Levi's et le recul de la mobilisation syndicale durant cette période d'austérité ont fait de la stratégie déployée par les ouvrières un cas atypique et isolé. À l'inverse de la cellule de l'emploi chez les sidérurgistes d'Athus, leur initiative n'a pas fait école et reste encore aujourd'hui mal connue.

\section{Contre-propositions ouvrières et paradigmes économiques}

Les contre-propositions ouvrières que nous venons de présenter nous semblent illustrer l'évolution des paradigmes économiques depuis la fin des années 1950 jusqu'au milieu des années 1980. Il semble, en effet, qu'en l'absence de perspectives de reclassement crédibles, l'analyse de l'évolution des activités industrielles régionales par les ouvriers subissant une restructuration converge avec l'émergence d'un discours économique dominant. Ni doctrine, ni théorie arrêtée, un paradigme économique peut être assimilé à un effet de mode ou, pour supprimer la connotation péjorative, à une modification des interprétations habituelles du fonctionnement de l'économie à une période historique donnée. La notion s'inspire des travaux de Thomas KuHN (1962) sur l'évolution de la pensée scientifique; Kuhn y définit le «paradigme» comme un consensus partagé par les chercheurs autour d'une théorie scientifique. À la différence de la démonstration de Kuhn, notre recherche ne tente pas d'étudier la succession des paradigmes économiques de 1959 à 1984 mais d'observer comment les ouvriers et ouvrières en sont venus à proposer une nouvelle lecture des transformations industrielles et à l'instrumentaliser dans le cadre de leur quête de reclassement professionnel. Le renouveau paradigmatique est en ce sens une opportunité pour surmonter une crise sectorielle profonde et un outil dans la lutte sociale pour que la reconversion économique s'accorde avec le reclassement du personnel licencié. Pour paraphraser la métaphore artistique

(35) Centre d'animation et de recherche en histoire ouvrière et populaire, archives de la CSC du Luxembourg conservées à Ciney, boîte 320, Procès-verbal de la réunion Idélux-ASBL et des responsables Ferrero, 23 novembre 1989. utilisée par Charles Tilly (1984), si la musique de ces stratégies ouvrières est commune, les paroles en sont modifiées au gré de la mise à l'agenda de nouvelles prédications économiques.

Précisément, le milieu des années 1970 marque une césure dans l'histoire économique de la deuxième partie du $\mathrm{Xx}^{\mathrm{e}}$ siècle. Décennie décrite comme une période charnière entre un avant et un après, c'est un no man's land affublé des préfixes pré- ou post- (Schulman, 2001; Chassaigne, 2008; KaElBLE, 2004). De nouvelles interprétations apparaissent alors pour définir, et dans une certaine mesure, pour légitimer les transformations qui agitent les sociétés occidentales. Les références à la société postindustrielle et au développement d'une économie de services symbolisent ce bouleversement paradigmatique (Graf, Priemel, 2011). Les travaux du sociologue Daniel Bell (1976) ou de l'économiste Jean FourastiÉ (1979) servent de cadre analytique et prospectif pour acter de la transition du travail manuel vers le travail intellectuel et pour offrir une explication scientifique à la fin des Trente Glorieuses.

Les stratégies déployées par les ouvriers et ouvrières dans les quatre cas de figure envisagés dans cet article ne relèvent pas d'initiatives extérieures et ont été élaborées empiriquement au gré des circonstances. Pour autant, il ne faudrait pas réduire ces contre-propositions aux situations sociales et économiques spécifiques dans lesquelles elles s'inscrivent. Un survol de quelques autres exemples de restructurations en Wallonie montre que ce type de stratégie a également parfois été mis en œuvre, le plus souvent avec l'appui des syndicats. Dans les quatre cas considérés ici, les structures syndicales nationales semblent certes peu motrices. Mais les organisations syndicales apprivoisent progressivement les méthodes et techniques de soutien à la reconversion du personnel. Ainsi, chez les ouvriers des soieries synthétiques de Fabelta en 1981, les syndicalistes FGTB et CSC présents dans la cellule de l'emploi montent une structure «création d'entreprises» pour soutenir les ouvriers licenciés qui élaborent un projet de reconversion. La participation syndicale à la formation et à la reconversion $\mathrm{du}$ personnel est loin d'avoir été systématique mais elle a connu quelques épisodes mémorables en Belgique, notamment lors de la gestion de la fermeture des Laminoirs de Jemappes ou de la restructuration de la clinique Cavell à Bruxelles en 1982. Pour la première fois dans l'histoire de la Belgique, les organisations syndicales deviennent un acteur central de la création d'entreprises et de la reconversion individuelle, et non plus seulement du reclassement (TiLly, 2007); par la suite, ils se sont investis fortement dans ce rôle d'accompagnateur 
social (36). Toutefois, la spécificité de nos quatre cas d'étude envisagés réside dans leur rapport à l'évolution des paradigmes économiques pour fonder leur contre-proposition ouvrière de reconversion.

Lorsque survient la crise charbonnière de 1958, les syndicats ne considèrent pas à l'époque qu'accompagner les ouvriers dans leurs trajectoires de reconversion professionnelle fait partie de leurs missions. L'enjeu consiste alors surtout à tenter de maintenir de l'activité dans les anciennes régions industrielles wallonnes sur fond de tensions économiques et politiques entre le Nord et le Sud du pays. Dans ce contexte, la Haute Autorité de la Ceca a joué un rôle moteur dans le revirement face au principe économique selon lequel c'était l'implantation des entreprises qui devait déterminer le déplacement des populations et non l'inverse. Il est possible d'identifier l'articulation entre le discours tenu sur la nécessité d'une relance industrielle régionale et les revendications des mineurs dans le cadre du déclin charbonnier. Les organisations syndicales ont très rapidement repris ce discours, soutenu par la population boraine et les élites politiques locales. La création d'immenses parcs industriels au début des années 1960 témoigne de l'engouement pour une régénération des activités productives autres que liées au charbon. Toutefois, malgré de multiples tentatives historiographiques, l'évaluation quantitative de la reconversion des mineurs demeure très approximative ${ }^{(37)}$.

Dans la seconde moitié des années 1970, les ouvrières de Siemens sont loin d'être les seules à constater la disparition de l'industrie manufacturière en Wallonie. À vrai dire, l'ensemble des luttes ouvrières au cours de cette période peut se résumer en une crise de l'avenir et de l'identité ouvrière wallonne. Mais la volonté des ouvrières de Siemens de se reconvertir collectivement dans le secteur tertiaire ne se retrouve dans aucun autre conflit social de l'époque. Comme dans le cas des huit sidérurgistes d'Athus, les stratégies ouvrières mises en œuvre montrent que là où les conditions le permettent, le changement de paradigme économique peut être un moteur du reclassement ouvrier. La prise d'initiative des ouvriers renverse les rôles

(36) Des sociologues belges travaillent actuellement sur le rôle des syndicats dans les cellules de reconversion, $c f$. BINGEN A. (2008), Le rôle de l'accompagnateur social au sein des cellules de reconversion wallonnes, Rapport de recherche pour la FGTB wallonne dans le cadre du projet européen Paros (Professionnaliser l'accompagnement des reconversions par les organisations syndicales); Bingen A., Cortese V., Stroobants M. (2008), Les modalités d'accompagnement des travailleurs licenciés collectivement, Rapport de recherche réalisé pour l'Institut wallon pour l'évaluation, la prospective et la statistique (Iweps); BINGEN A. (2012), Licenciements collectifs et reconversion de la main-d' 'euvre. Le cas des cellules de reconversion wallonnes, Thèse de doctorat soutenue à l'Université libre de Bruxelles.

(37) Sur 4059 emplois créés dans le bassin du Borinage dans les années 1960 et sur lesquels une étude a été menée, seulement $10 \%$ de ces emplois sont occupés par d'anciens mineurs. classiquement attribués au sein du dialogue social belge et souligne la responsabilité des pouvoirs publics et des employeurs. Ceci expliquerait le succès manifeste des tentatives d'autoproduction en Belgique où la substitution de l'ouvrier à l'employeur est tour à tour perçue comme une microrévolution ou comme une démarche pragmatique de maintien de l'outil productif dans l'attente d'un repreneur.

Comme mentionné plus haut, c'est peut-être parce que la production chez Siemens était destinée au secteur administratif que les ouvrières ont cru que le travail de bureau pouvait constituer une échappatoire aux restructurations qui frappaient le secteur manufacturier. La présence d'intellectuels, d'artistes et de militants de la Nouvelle Gauche lors de l'occupation de l'usine est peut-être aussi un facteur explicatif de leur engagement dans cette voie (HemmeriJckx, 2007). Plusieurs syndicalistes issus de régions à l'industrialisation récente prennent en effet part à la lutte chez Siemens, et dans une certaine mesure, influencent la forme prise par le conflit. Plus jeunes et plus indépendants, ils sont plus ouverts aux nouvelles idées et stratégies de lutte. Cette mobilisation pour le reclassement collectif dans le secteur tertiaire s'avère finalement être un échec en raison des qualifications insuffisantes des ouvrières. Mais le conflit Siemens a d'autres conséquences dans la mesure où il ouvre la voie à la contestation ouvrière féminine qui prend des formes aussi diverses que les événements du Balai libéré en $1975^{(38)}$ ou l'autoproduction par les ouvrières chez Daphica ${ }^{(39)}$.

Les pistes qui mènent certains sidérurgistes de l'acier à la logique européenne du marché commun sont plus faciles à suivre. Des responsables de la reconversion régionale rappellent combien le marché commun et la position de carrefour européen de la Province du Luxembourg peuvent être des éléments moteurs d'une relance économique pour Athus et ses ouvriers ${ }^{(40)}$. La campagne de communication visant à attirer les investisseurs étrangers cible justement son argumentation autour de la centralité européenne de la région, au cœur de l'ancien croissant prospère qui s'étirait des PaysBas à la Toscane. Dans les faits, le bassin d'emplois est saturé pour les sidérurgistes et la probabilité de pouvoir les reclasser à moyen terme dans des entreprises implantées ultérieurement, semble bien

(38) Le Balai libéré est une entreprise de nettoyage à Louvainla-Neuve, en autogestion, lancée en 1975 après le licenciement symbolique du patron par les ouvrières.

(39) La reprise en main par les ouvrières de l'usine de laine Daphica à Tournai déclarée en faillite en 1974 représente la réussite la plus accomplie d'une coopérative de production ouvrière de cette période.

(40) Entretien avec Daniel Gheza, comptable à la Société de diversification du bassin belgo-luxembourgeois (SDBL) et employé à l'intercommunale Idélux, 18 avril 2012. Entretien avec Henri Demortier, employé à l'intercommunale Idélux, 3 mars 2011. Tous deux ont contribué à la politique de relance économique du Sud-Luxembourg au lendemain de la fermeture de l'usine d'Athus. 
faible. L'expérience professionnelle de certains cadres et ouvriers du service traction de la société leur a permis de concevoir une opportunité de reclassement. Le discours tenu par les institutions européennes lors de la crise sidérurgique des années 1980 et la perspective d'un Pôle européen de développement alimentent le potentiel de réussite du projet. L'idée d'un marché commun producteur de richesse a en outre l'avantage de replacer Athus au centre d'un appareil productif plus large, souhaité par les hommes politiques locaux, à commencer par Jacques Planchard, gouverneur de la Province du Luxembourg, ancien fonctionnaire en charge de la politique territoriale de la Ceca et instigateur du plan de relance économique de la région. Le slogan «faire de trois culs-de-sac nationaux un centre européen» a parfaitement été assimilé par les anciens sidérurgistes. Cette initiative serait-elle un cas isolé de tentative de relance collective d'une activité industrielle ? Des événements similaires se sont pourtant produits à l'étranger (41).

Si des perspectives nouvelles ont pu être théoriquement proposées aux mineurs du Borinage, aux ouvrières de Siemens ainsi qu'aux sidérurgistes d'Athus, rien de tel pour les couturières de jeans Levi's d'Arlon. Ce "rien» leur sert en quelque sorte de moteur pour prendre la main et développer leur propre initiative en l'absence de soutien des syndicats et des autorités locales. Les fonds publics importants disponibles pour les sidérurgistes et les mineurs ne le sont plus pour ces ouvrières dont les salaires sont en fin de compte encore considérés comme des salaires d'appoint.

$\mathrm{Au}$ cœur de cette décennie marquée par les gouvernements Martens-Gol inspirés des politiques reaganiennes et thatchériennes, le mouvement ouvrier belge est en net repli et en panne d'inspiration. C'est de cette absence totale de perspective de reclassement que surgit l'idée d'utiliser l'argent du fonds de licenciement afin que la force du nombre leur permette de devenir des acteurs économiques disposant d'une capacité d'influence. Le résultat n'est pas négligeable, bien que tardif puisque une centaine d'ouvrières de Levi's est reclassée dans la nouvelle implantation Ferrero.

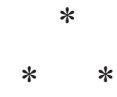

Un quart de siècle sépare la mobilisation des mineurs du Borinage de celle des couturières d'Arlon. En vingt-cinq ans, les ouvriers et ouvrières confrontés aux restructurations de leur secteur industriel ont développé des stratégies variées et inédites pour les surmonter. Les quatre cas décrits dans l'article ne sont pas représentatifs de l'ensemble des stratégies mises en œuvre en Wallonie face à des restructurations et ne constituent pas des «modèles» des réponses ouvrières auxquelles elles ont donné lieu. Les propositions de reconversion initiées par les ouvriers s'inscrivent dans des contextes économiques, sociaux et politiques variés tout en témoignant de fortes singularités. Ainsi, la constitution d'une association avec l'utilisation d'une partie des indemnités de licenciement en guise d'appât pour un investisseur ou la tentative de requalification collective vers un emploi de bureau sont insolites dans le dispositif contestataire des ouvriers en Wallonie.

De ce parcours exploratoire des contre-propositions ouvrières aux restructurations, de nombreuses zones d'ombre nécessiteraient des investigations complémentaires. Au vu du rôle primordial des organisations syndicales dans le système politique et le dialogue social belges, il n'est pas étonnant qu'elles aient pris part activement au processus de reconversion. Mais dans la mesure où leur participation n'a été ni linéaire, ni systématique, il conviendrait d'observer quel rôle jouent la mise en place de structures et l'intervention d'experts spécialisés dans l'émergence de ces contre-propositions ouvrières. Le rôle de la «Form'Action André Renard» pour la sidérurgie (LomBA, 2013) ou de l'association «Aide à la reconversion» dans la région de Charleroi par l'insertion professionnelle et la création d'emplois locaux (Archipel) constitueraient des pistes pertinentes d'autant plus que ces structures sont conçues dans une perspective collective de réponse à la situation des sans-emploi. Dans un second temps, il s'agirait d'examiner les différentes dimensions de ces contre-propositions ouvrières : des plus immédiates (autoproduction pour entretenir l'outil productif et quête d'un repreneur) aux plus insolites et utopiques (création d'un circuit commercial autogestionnaire parallèle au circuit traditionnel). Dans tous les cas de figure, la démarche consisterait à dépasser les effets de mode, tels que la vague autogestionnaire, pour accéder à une parole ouvrière qui associerait vécus au travail, expériences de la restructuration et projections pour un avenir incertain. 


\section{Bibliographie}

Ait Oumeziane A. (2000), «Reconversion économique et construction d'un territoire transfrontalier : l'agglomération transfrontalière du Pôle européen de développement des trois frontières (Belgique-FranceLuxembourg)», Annales de géographie, $\mathrm{n}^{\circ}$ 611, pp. 65-83.

ANDRÉ R. (1972), «L'impact démographique de la crise dans une commune du Borinage, Flénu 1955-1971», Revue de l'Institut de sociologie, $\mathrm{n}^{\circ} 2$, pp. 319-354.

Azzaoui B., Noiriel G. (1980), Vivre et lutter à Longwy, Paris, Maspero.

BASTIN J.-C. (1977), Les travailleurs de la MMRA. Potentiel de main-d'ouvre et attitudes à l'égard de la formation et $d u$ reclassement, Rapport de recherche, Fondation universitaire du Luxembourg.

Beatty C., Fothergill S. (1996), « Labour market adjustment in areas of chronic decline: the case of the UK coalfields », Regional Studies, vol. 30, n 7, pp. 627-640.

Bell D. (1976), The coming of postindustrial society: a venture in social forecasting, New York, Basic Books.

Bettens L., Geerkens É. (2010), «Des occupations d'usine à la médiation culturelle», in Delhalle N., Dubois J. (dir.), Le tournant des années 1970, Liège en effervescence, Bruxelles, Les Impressions nouvelles, pp. 63-82.

Bingen A. (2008), Le rôle de l'accompagnateur social au sein des cellules de reconversion wallonnes, Rapport de recherche pour la FGTB wallonne dans le cadre du projet européen Paros (Professionnaliser l'accompagnement des reconversions par les organisations syndicales).

Bingen A., Cortese V., Stroobants M. (2008), Les modalités d'accompagnement des travailleurs licenciés collectivement, Rapport de recherche réalisé pour l'Institut wallon pour l'évaluation, la prospective et la statistique (Iweps).

BINGENA. (2012), Licenciements collectifs et reconversion de la main-d'œuvre. Le cas des cellules de reconversion wallonnes, Thèse de doctorat soutenue à l'Université libre de Bruxelles.

Bruwier M. (1996), «Connaissance historique de la zone de reconversion (l'arrondissement de Mons)», in Industrie et société en Hainaut et en Wallonie du XVII au XXe siècle : recueil d'articles de Marinette Bruwier, Bruxelles, Crédit communal, pp. 385-394.

Cammarata F., Tilly P. (2001), Histoire sociale et industrielle de la Wallonie, Bruxelles, EVO.

CAPron M. (1977), «Siemens, une multinationale, l'État... et des travailleuses», La Revue nouvelle, mai-juin, pp. 491-492.

Charasse D. (1980), Lorraine Cour d'acier, Paris, Maspero.
Chassaigne P. (2008), Les années 1970 : fin d'un monde et origine de notre modernité, Paris, Armand Colin.

Clark J. (2011), « Closing Moulinex: thoughts on the visibility and invisibility of industrial labour in contemporary France ", Modern \& Contemporary France, vol. 19, n 4, pp. 443-458.

Corteel D. (2009), «Fermetures d'usines: les associations d'anciens salariés comme espaces de médiation originaux», Formation emploi, $\mathrm{n}^{\circ} 108$, pp. 53-65.

Degee J.-L. (1980), L'évolution des luttes ouvrières en Belgique, Liège, Fondation André-Renard.

Deshayes J.-L. (2010), La conversion territoriale, Longwy (1978-2010). Le salariat entre paternalisme et mondialisation, Nancy, Presses universitaires de Nancy.

Evalenko R. (1968), Régime économique de la Belgique, Bruxelles-Louvain, Vander.

FAVIER I. (2009), «Les restructurations à l'usine Perrier, ou quand deux “cultures d'entreprise" s'affrontent sur le lieu de travail (1990-2000)», Mouvement social, n²28, pp. 147-162.

Fieldhouse E., Hollywood E. (1999), « Life after mining: hidden unemployment and changing patterns of economic activity amongst miners in England and Wales, 1981-1991 », Work, employment and society, vol. 13, $\mathrm{n}^{\circ} 2$, pp. 483-502.

Fourastié J. (1979), Les Trente Glorieuses, ou la révolution invisible de 1946 à 1975, Paris, Fayard.

Goffin L., Mormont M., Tibesar A. (1981), «La fermeture de l'usine d'Athus : analyse des conséquences économiques, effets des mesures sociales et de la politique de reconversion », Courrier hebdomadaire $d u$ Crisp, $\mathrm{n}^{\circ}$ 935, pp. 1-72.

Graf R., Priemel K. (2011), «Zeitgeschichte in der Welt der Sozialwissenschaften Legitimität und Originalität einer Disziplin», Vierteljahrshefte für Zeitgeschichte, $\mathrm{n}^{\circ} 4$, pp. 479-508.

GubBels R. (1962), La grève, phénomène de civilisation, Bruxelles, Institut de sociologie-Université libre de Bruxelles.

Hayes I. (2013), «Les limites d'une médiation militante. L'expérience de Radio Lorraine Cœur d'acier, Longwy, 1979-1980», Actes de la recherche en sciences sociales, $\mathrm{n}^{\circ}$ 196-197, pp. 84-101.

Hegale M., Layon E. (2002), «De l'employabilité à la convertibilité : vers une mobilité offensive des travailleurs », Revue du TEF, $\mathrm{n}^{\circ}$ 3, pp. 15-25.

Hemmerijckx R. (2007), «In de geest van Mei 68. Arbeidersprotest en radicaal militantisme in België», Cahiers d'histoire du temps présent, $\mathrm{n}^{\circ} 18, \mathrm{pp}$. 163-182. 
Hobsbawm E. J. (1952), « The machine breakers », Past and Present, $\mathrm{n}^{\circ} 1, \mathrm{pp}$. 57-70.

Houseman S. (1991), Industrial restructuring with job security. The case of European steel, Cambridge, Harvard University Press.

HudSON R. (1994), « Institutional change, cultural transformation and economic regeneration: myths and realities from Europe's old industrial areas », in Amin A., Thrift N. (dir.), Globalization, institution, and regional development in Europe, Oxford, Oxford University Press, pp. 196-216.

Jahoda M., Lazarsfeld P., Zeisel H. (1982), Les chômeurs de Marienthal, Paris, Éditions de Minuit.

JARRIDGE F. (2009), Au temps des «tueuses de bras». Les bris de machines à l'aube de l'ère industrielle, Rennes, Presses universitaires de Rennes.

Kaelble H. (2004), «Vers une histoire sociale et culturelle de l'Europe pendant les années de l'après-prospérité», Vingtième siècle. Revue d'histoire, octobre-décembre, n 84, pp. 169-179.

Kunn T. (1962), The structure of scientific revolutions, Chicago, University of Chicago Press.

Lahaye F. (2005), «Professionnels du textile: se construire une conscience fière», Ethnologie française, vol. $35, \mathrm{n}^{\circ} 4$, pp. 703-713.

Leboutte R. (2008), Histoire économique et sociale de la construction européenne, Bern-Bruxelles, Peter Lang.

Le Quentrec Y., Benson S. (2005), Un job pour la vie, les salariés de Job en lutte (1995-2001), Paris, Syllepse.

Lomвa C. (2001), L’incertitude stratégique au quotidien. Trajectoire d'entreprise et pratiques de travail. Le cas de l'entreprise sidérurgique Cockerill Sambre, 1970-1998, Thèse pour le doctorat de sociologie, EHESS.

LombA C. (2013), «Restructurations industrielles : appropriations et expropriations des savoirs ouvriers ", Actes de la recherche en sciences sociales, $\mathrm{n}^{\circ} 196-197$, pp. 34-53.

Maruani M. (1979), Les syndicats à l'épreuve du féminisme, Paris, Syros.

Mazade O. (2010), La reconversion des hommes et des territoires : le cas Metaleurop, Paris, L'Harmattan.

MéNY Y., Wright V. (dir) (1985), La crise de la sidérurgie européenne, 1974-1984, Paris, Presses universitaires de France.

Milward A. (1992), The European rescue of the nationstate, Londres, Routledge.
Minon P., Demet F. (1956), «Migrations provoquées et problèmes sociaux de mobilité ouvrière», Travaux de l'Institut de sociologie de la faculté de droit de Liège.

Molitor M. (1978), « Social conflicts in Belgium », in Crouch C., Pizzorno A. (dir.), The resurgence of class conflict in Western Europe since 1968, Londres, Macmillan, vol. 1, pp. 21-51.

Morelli A. (1988), «L'appel à la main-d'œuvre italienne dans les charbonnages et sa prise en charge à son arrivée en Belgique dans l'immédiat après-guerre», Revue belge d'histoire contemporaine, $\mathrm{n}^{\circ}$ 1-2, pp. 83-130.

Perron R. (1996), Le marché du charbon, un enjeu entre l'Europe et les États-Unis de 1945 à 1958, Paris, Publications de la Sorbonne.

ReID D. (1999), The miners of Decazeville. A genealogy of deindustrialization, New York, toExcel, $2^{\text {nd }}$ edition.

Rostow W. W. (1962), The stages of economic growth, Londres, Cambridge University Press.

Roupnel-Fuentes M. (2011), Les chômeurs de Moulinex, Paris, Presses universitaires de France.

SChulman B. (2001), The seventies: the great shift in American culture, society and politics, Cambridge, Da Capo Press.

Thompson E. (1971), « The moral economy of the English crowd in the eighteenth century », Past and Present, $\mathrm{n}^{\circ} 50$, pp. $76-136$.

Tilly C. (1984), «Les origines de l'action collective contemporaine en France et en Grande-Bretagne», Vingtième siècle. Revue d'histoire, n 4, pp. 89-108.

Tilly P. (2007), Origines et évolutions des politiques et des actions d'accompagnement des reconversions en Wallonie de 1977 à 2006, Cahiers Fopes, Louvain-laNeuve, Presses universitaires de Louvain.

Verschueren N. (2011), «L'expression culturelle de la contestation dans un ancien bassin minier», Mouvements, $\mathrm{n}^{\circ} 65$, pp. 67-78.

Verschueren N. (2013a), Fermer les mines en construisant l'Europe. Une histoire sociale de l'intégration européenne, Bruxelles, Peter Lang.

VERSCHUEREN N. (2013b), «Fermeture et reconversion aux frontières : un univers postindustriel ? Le cas d'Athus "), Mutations. Mémoires et perspectives du bassin minier, $\mathrm{n}^{\circ} 6$, pp. 27-38.

Vigna X. (2004), «Les ouvriers de Denain et de Longwy face aux licenciements (1978-1979), Vingtième siècle. Revue d'histoire, vol. 84, n 4, pp. 129-137. 